H. Ichimura

Nagoya Math. J.

Vol. 99 (1985), 63-71

\title{
A NOTE ON QUADRATIC FIELDS IN WHICH A FIXED PRIME NUMBER SPLITS COMPLETELY
}

\author{
HUMIO ICHIMURA
}

\section{§1. Introduction}

Throughout this note, $p$ denotes a fixed prime number and $f$ denotes a fixed natural number prime to $p$.

It is easy to see and more or less known that ${ }^{(*)}$ for any natural number $n$, there exists an elliptic curve over $\bar{F}_{p}$ whose $j$-invariant is of degree $n$ over $\boldsymbol{F}_{p}$ and whose endomorphism ring is isomorphic to an order of an imaginary quadratic field. In this note, we consider a more precise problem: for any natural number $n$, decide whether or not there exists an elliptic curve over $\overline{\boldsymbol{F}}_{p}$ whose $j$-invariant is of degree $n$ over $\boldsymbol{F}_{p}$ and whose endomorphism ring is isomorphic to an order of an imaginary quadratic field with conductor $f$.

To state our results, we introduce some notations. For an order $\mathfrak{o}$ of a quadratic field $K$, we write $(\mathfrak{o} / p)=1$ when $(K / p)=1$ and the conductor of $\mathfrak{o}$ is prime to $p$, where $(K / p)$ denotes the Legendre symbol. Let $\mathfrak{P}$ be a prime divisor of $p$ in $\overline{\boldsymbol{Q}}$. For an order $\mathfrak{o}$ of a quadratic field with (o/p) $=1$, we set $\mathfrak{p}_{\mathfrak{o}}=\mathfrak{P} \cap \mathfrak{o}$ and we denote by $n_{\mathfrak{o}}$ the number of elements of the cyclic subgroup of the proper 0 -ideal class group generated by the proper o-ideal class $\left\{\mathfrak{p}_{0}\right\}$. Clearly, $n_{0}$ does not depend on the choice of $\mathfrak{P}$.

Set $M(p, f)=\{\mathfrak{o}$; orders of imaginary quadratic fields with $(\mathfrak{o} / p)=1$ and conductor $f\}$. Let $N(p, f)$ be the image of the map $M(p, f) \ni \mathfrak{o} \rightarrow n_{\circ}$ $\in N$.

By some results of Deuring on elliptic curves (see e.g. Lang [6]; Chap. 13, Theorem 11, 12, and Chap. 14, Theorem 1), the preceding problem is equivalent to a problem: decide the image $N(p, f)$.

Our results are as follows.

TheOREM 1. (i) When $(p / l)=1$ for any odd prime divisor $l$ of $f$, and

(*) We give a simple proof in Remark 1 of $\S 4$. 
$8 \nmid f($ resp. $4 \nmid f)$ in the case $p \equiv 5(\bmod 8)($ resp. $p \equiv 3(\bmod 4))$, the complement $N-N(p, f)$ is a finite set, (ii) otherwise, $N(p, f) \subset 2 N$, and the complement $2 N-N(p, f)$ is a finite set.

Theorem 2. $N(p, 1)=N$.

Further, for real quadratic fields, we show a fact similar to (but not as sharp as) Theorem 1, 2.

Ankeny and Chowla [1] proved $|N-N(3,1)|<\infty$ (a special case of Theorem 1). For a fixed natural number $n$, set $m(p, n)=\mid\{\mathfrak{0} \in M(p, 1)$; $\left.n_{0}=n\right\} \mid$. Humbert [4] and Kuroda [5] proved that $m(p, n) \rightarrow \infty$ as $p \rightarrow \infty$. By these facts, they showed the existence of infinitely many imaginary quadratic fields with class number divisible by a given integer. Theorem 1 is proved by using the method of [4], [1] and [5]. To prove Theorem 2, we first calculate a number $n_{p}$ such that $n \in N(p, 1)$ if $n \geq n_{p}$, with the help of an approximation formula of Rosser and Schoenfeld [8] for $\pi(x)$, the number of prime numbers $\leq x$. Next, we construct orders $\mathfrak{v} \in M(p, 1)$ with $n_{0}=n$ for "small" $n$ explicitly.

Notations. $\quad \boldsymbol{N}, \boldsymbol{Z}, \boldsymbol{Q}$ and $\boldsymbol{F}_{p}$ denote, respectively, the set of natural numbers, the ring of rational integers, the field of rational numbers and the finite field with $p$ elements. For a field $K, \bar{K}$ denotes the algebraic closure of $K$. For an element $a$ of a quadratic field, $a^{\prime}$ and $N(a)$ denotes its conjugate and its norm respectively.

\section{§. Proof of Theorem 1}

Let $p$ be a fixed prime number and $f$ a fixed natural number prime to $p$. There are two possible cases.

[I] $(p / l)=1$ for any odd prime divisor $l$ of $f$, and $8 \nmid f$ (resp. $4 \nmid f$ ) in the case $p \equiv 5(\bmod 8)(\operatorname{resp} . p \equiv 3(\bmod 4))$,

[II] otherwise.

First, we show the following

Lemma 1. In case [II], $N(p, f) \subset 2 N$.

Proof. The condition [II] means that $(p / l)=-1$ for some odd prime divisor $l$ of $f$, or $8 \mid f$ and $p \equiv 5(\bmod 8)$, or $4 \mid f$ and $p \equiv 3(\bmod 4)$. Let $\mathfrak{D}$ be an order of an imaginary quadratic field with $(\mathfrak{o} / p)=1$ and conductor $f$. Let $d$ be the discriminant of the imaginary quadratic field $\mathfrak{o} \otimes_{Z} \boldsymbol{Q}$. First, assume that $(p / l)=-1$ for some odd prime divisor $l$ of $f$ and $d \equiv 0$ 
$(\bmod 4) . \quad$ Then, $\mathfrak{o}=[1, f \sqrt{d / 4}] . \quad$ By the definition of $n_{\mathrm{o}}, \mathfrak{p}_{\mathfrak{o}}^{n_{0}}=(a+b f \sqrt{d / 4})$ for some $a, b \in Z$. Taking norms of both sides, $p^{n_{0}}=a^{2}-b^{2} f^{2}(d / 4)$. Therefore, if $n_{\mathrm{o}}$ is odd, $(p / l)=1$ for any odd prime divisor $l$ of $f$, which is a contradiction. So, $n_{0}$ must be even. It is proved similarly in the other cases.

Now, we prove that $N-N(p, f)$ (resp. $2 N-N(p, f)$ ) is a finite set in case [I] (resp. [II]). First, we deal with the case where $f$ is odd and satisfying the condition [I].

The following lemma is easily proved.

Lemma 2. Assume $f$ is odd. Let $n$ be a natural number, and let $x$ be a rational integer, prime to $2 p$ and satisfying the following conditions:

(i) $x^{2} \equiv 4 p^{n}\left(\bmod f^{2}\right)$

(ii) $\frac{x^{2}-4 p^{n}}{f^{2}}$ is square free,

(iii) $0<x<2 \sqrt{p^{n}-p^{n / 2}}$.

Let $\mathfrak{D}$ be the order the imaginary quadratic field $K=Q\left(\sqrt{x^{2}-4 p^{n}}\right)$ with conductor $f$. Then, $(\mathfrak{o} / p)=1$ and $n_{0}=n$.

Let $f=\prod_{i} l_{i}^{e_{i}}$ be the prime decomposition of $f$, and set $f_{0}=\prod_{i} l_{i}$. Since $f$ is odd and satisfies the condition [I], there exists an odd integer $x(n)$ such that $x(n)^{2} \equiv 4 p^{n}\left(\bmod f^{2}\right)$ and $x(n)^{2} \not \equiv 4 p^{n}\left(\bmod l^{2} f^{2}\right)$ for any prime divisor $l$ of $f$. Set $A(n)=\left\{x(n)+2 f_{0}^{2} f^{2} k ; k \in Z\right\}$ and $B(n)=\{x \in A(n) ; x$ is prime to $p, x^{2} \not \equiv 4 p^{n}\left(\bmod l^{2}\right)$ for any odd prime number $l$ with $l \nmid f$, and $\left.0<x<2 \sqrt{p^{n}-p^{n / 2}}\right\}$. By Lemma 2, it suffices to show that $|B(n)| \rightarrow \infty$ as $n \rightarrow \infty$. The number of $x \in A(n)$ such that $x$ is prime to $p$ and $0<x$ $<2 \sqrt{p^{n}-p^{n / 2}}$ is at least $\left[(1-1 / p)\left(\left(\sqrt{p^{n}-p^{n / 2}}\right) / f_{0}^{2} f^{2}\right)\right]-2$ if $p \neq 2$, and $\left[\left(\sqrt{p^{n}-p^{n / 2}}\right) / f_{0}^{2} f^{2}\right]$ if $p=2$, where $[a]$ denotes the largest integer $\leq a$.

Let $l$ be an odd prime number with $l \nmid p f$. Since the congruence $x^{2} \equiv$ $4 p^{n}\left(\bmod l^{2}\right)$ has at most two solutions, the number of $x \in A(n)$ such that $x^{2} \equiv 4 p^{n}\left(\bmod l^{2}\right)$ and $0<x<2 \sqrt{p^{n}-p^{n / 2}}$ is at most $2\left\{\left[\left(\sqrt{p^{n}-p^{n / 2}}\right) / f_{0}^{2} f^{2} l^{2}\right]\right.$ $+1\}$ if $l<2 p^{n / 2}$, and is zero if $l \geq 2 p^{n / 2}$.

Therefore,

$$
|B(n)|>\left\{\begin{array}{l}
{\left[\left(1-\frac{1}{p}\right) \frac{\sqrt{p^{n}-p^{n / 2}}}{f_{0}^{2} f^{2}}\right]-2-\sum_{l}^{\prime}\left\{2\left[\frac{\sqrt{p^{n}-p^{n / 2}}}{f_{0}^{2} f^{2} l^{2}}\right]+2\right\}} \\
\text { if } p \neq 2 \\
{\left[\frac{\sqrt{p^{n}-p^{n / 2}}}{f_{0}^{2} f^{2}}\right]-\sum_{l}^{\prime}\left\{2\left[\frac{\sqrt{p^{n}-p^{n / 2}}}{f_{0}^{2} f^{2} l^{2}}\right]+2\right\} \quad \text { if } p=2}
\end{array}\right.
$$




$$
>\left\{\begin{array}{l}
\frac{1}{f_{0}^{2} f^{2}}\left\{\left(1-\frac{1}{p}\right)-2 \sum^{\prime} \frac{1}{l^{2}}\right\} \sqrt{p^{n}-p^{n / 2}}-3-2 \sum_{l}^{\prime \prime} 1 \text { if } p \neq 2 \\
\frac{1}{f_{0}^{2} f^{2}}\left\{1-2 \sum_{l}^{\prime}\right\} \sqrt{p^{n}-p^{n / 2}}-1-2 \sum_{l}^{\prime \prime} 1 \text { if } p=2,
\end{array}\right.
$$

where the sum $\sum_{l}^{\prime}$ is taken over all prime numbers $l$ prime to $2 p f$ with $0<l<2 p^{n / 2}$, and the sum $\sum_{l}^{\prime \prime}$ is taken over all prime numbers $l$ with $0<l<2 p^{n / 2}$.

Note that $\sum_{l}^{\prime} 1 / l^{2}<\log \zeta(2)-1 / 4-1 / p^{2}($ resp. $\log \zeta(2)-1 / 4)$ when $p \neq 2$ (resp. $p=2$ ), where $\zeta(s)$ is the Riemann zeta function. Therefore, by $\zeta(2)$ $=\pi^{2} / 6$, we see that the coefficient of $\sqrt{p^{n}-p^{n / 2}}$ is larger than the positive constant $c_{p} / f_{0}^{2} f^{2}$, where $c_{p}$ is the positive constant given as follows:

(Table 1)

\begin{tabular}{c|c|c|c|c|c}
\hline$p$ & $p \geq 11$ & 7 & 5 & 3 & 2 \\
\hline$c_{p}$ & 0.429 & 0.401 & 0.384 & 0.392 & 0.504 \\
\hline
\end{tabular}

On the other hand, by the prime number theorem,

$$
\sum_{i}^{\prime \prime} 1=O\left(\frac{2 p^{n / 2}}{(n / 2) \log p}\right)
$$

Therefore, $|B(n)| \rightarrow \infty$ as $n \rightarrow \infty$. This completes the proof of Theorem 1 when $f$ is odd and satisfies the condition [I].

It is proved similarly in the other cases.

\section{§3. Proof of Theorem 2}

Let $\pi(x)$ be the number of prime numbers $\leq x$. Rosser and Schoenfeld [8] (Theorem 2) showed

$$
\pi(x)<\frac{x}{\log x-3 / 2} \quad \text { for } x>e^{3 / 2} .
$$

By a simple calculation using (1), (2) and Table 1, we obtain

Lemma 3. The set $N(p, 1)$ contains all natural numbers $n$ with $n \geq n_{p}$, where $n_{p}$ is the natural number given in the following table.

\begin{tabular}{c|c|c|c|c|c}
\hline$p$ & $p \geq 11$ & 7 & 5 & 3 & 2 \\
\hline$n_{p}$ & 10 & 12 & 16 & 21 & 26 \\
\hline
\end{tabular}


By this lemma, it suffices to construct orders $\mathfrak{v} \in M(p, 1)$ with $n_{\mathrm{o}}=n$ for "small" $n$.

LEMma 4. The set $N(p, 1)$ contains all natural numbers of the form $n=2^{\lambda} 3^{\mu} 5^{\nu} 7^{\chi}$ with $\lambda, \mu, \nu, \chi \geq 0$.

Proof. First, we prove our lemma when $p \neq 3$. Fix a natural number $k$ and set $m=p^{k}$. Set $\boldsymbol{K}_{1, l}=\boldsymbol{Q}\left(\sqrt{1-4 m^{i}}\right)$ and $\boldsymbol{K}_{2, l}=\boldsymbol{Q}\left(\sqrt{9-4 m^{i}}\right)$ for $l=1,2,3,5,7$. When $p \neq 3,\left(K_{i, l} / p\right)=1$ and we denote by $\mathfrak{p}_{i, l}$ a prime ideal $^{(*)}$ of $K_{i, l}$ over $p(i=1,2, l=1,2,3,5,7)$. We show

Claim 1. Assume $p \neq 3$. The ideal class ${ }^{(*)}$ of $\mathfrak{p}_{1,2}^{k}\left(\right.$ in $\left.K_{1,2}\right)$ or that of $\mathfrak{p}_{2,2}^{k}\left(\right.$ in $\left.K_{2,2}\right)$ is of order 2 .

This is proved as follows. Write $1-4 m^{2}=f_{1}^{2} d_{1}$ and $9-4 m^{2}=f_{2}^{2} d_{2}$ with natural numbers $f_{1}, f_{2}$ and square free integers $d_{1}, d_{2}$. Then, $d_{i} \equiv 1$ $(\bmod 4)$ and $1,\left(1+\sqrt{d_{i}}\right) / 2$ is an integral basis of $\boldsymbol{K}_{i, 2}$. Note that $\boldsymbol{K}_{i, 2} \neq$ $\boldsymbol{Q}(\sqrt{-1})$ because $d_{i} \equiv 1(\bmod 4)$. Set $\alpha_{1}=\left(1+\sqrt{1-4 m^{2}}\right) / 2$ and $\alpha_{2}=$ $\left(3+\sqrt{9-4 m^{2}}\right) / 2$. Then, we easily see that $\alpha_{i}$ is an integer of $\boldsymbol{K}_{i, 2},\left(\alpha_{i}, \alpha_{i}^{\prime}\right)$ $=1$ and $N\left(\alpha_{i}\right)=p^{2 k}$. Hence, we may assume, without loss of generality, that $\mathfrak{p}_{i, 2}^{2 k 1}=\left(\alpha_{i}\right)$. Assume that $\mathfrak{p}_{1,2}^{k}$ is principal. Then, since $K_{1,2} \neq \boldsymbol{Q}(\sqrt{-1})$, $\alpha_{1}= \pm\left(\left(a+b \sqrt{\left.d_{1}\right) / 2}\right)^{2}\right.$ for some $a, b \in Z$. Therefore, $1= \pm\left(a^{2}+b^{2} d_{1}\right) / 2$ and $f_{1}= \pm a b$. Hence, $1-4 m^{2}=f_{1}^{2} d_{1}=a^{2}\left( \pm 2-a^{2}\right)$, from which we obtain $2 m=a^{2} \pm 1$. By considering both sides modulo 4 , we see that $a$ is odd and $2 m=a^{2}+1$ (resp. $2 m=a^{2}-1$ ) when $m$ is odd (resp. even). Next, assume that $\mathfrak{p}_{2,2}^{k}$ is principal. Then, similarly, for some odd integer $c, 2 m$ $=c^{2}-3$ (resp. $2 m=c^{2}+3$ ) when $m$ is odd (resp. even). Therefore, if both of $\mathfrak{p}_{1,2}^{k}$ and $\mathfrak{p}_{2,2}^{k}$ are principal, $c^{2}=a^{2}+4$ for some odd integers $a$ and c. But this is impossible because the square of an odd integer is congruent to 1 modulo 8 . Hence, we obtain our claim. Similarly and more easily, we can prove

Claim $2^{(* *)}$. Assume $p \neq 3$. For $l=1,3,5,7$, the ideal class of $\mathfrak{p}_{\imath, l}^{k}$ is of order $l(i=1,2)$.

Now, set $n=2^{\lambda} 3^{\mu} 5^{\nu} 7^{\chi}$ with $\lambda, \mu, \nu, \chi \geq 0$. By the above claims, we see that for the maximal order 0 of the imaginary quadratic field $Q\left(\sqrt{1-4 p^{n}}\right)$

(*) In this section, an ideal (class) is one with respect to the maximal order of an imaginary quadratic field.

(**) Further, we can show that for any prime number $l(\geq 7)$, the ideal class of $p_{i, l}^{k}$ is of order $l$ for sufficiently large $p$. 
or that of $\boldsymbol{Q}\left(\sqrt{9-4 p^{n}}\right),(\mathfrak{o} / p)=1$ and $n_{\mathrm{o}}=n$. This proves our lemma when $p \neq 3$. When $p=3$, we can prove our lemma similarly by considering imaginary quadratic fields of type $K_{2, l}^{\prime}=Q\left(\sqrt{25-4 m^{l}}\right)$ in place of $K_{2, l}$.

Lemma 5. Assume $p$ is odd. Then, the set $N(p, 1)$ contains all odd natural numbers prime to $p$.

Proof. Let $n$ be an odd natural number prime to $p$. Let $n_{1}$ be the largest square free integer $\mid n$. Note that $n_{1}^{2}<p^{n}$. We easily see that for the maximal order $\mathfrak{v}$ of the imaginary quadratic field $\boldsymbol{Q}\left(\sqrt{n_{1}^{2}-p^{n}}\right),(\mathfrak{o} / p)$ $=1$ and $n_{\mathrm{v}}=n$, by the following

Theorem (Nagel [7], Satz V). Let $n$ be an odd natural number. Let $x$ and $z$ be natural numbers such that $(x, z)=1, x^{2}<z^{n}, 2 \nmid z$, and $q \| x$ for any prime divisor $q$ of $n$. Let $z=\prod_{i} q_{i}^{e_{i}}$ be the prime decomposition of z. Set $K=Q\left(\sqrt{x^{2}-z^{n}}\right)$. Then, $\left(K / q_{i}\right)=1$ and $\mathfrak{q}_{i}=\left(q_{i}, x+\sqrt{x^{2}-z^{n}}\right)$ is a prime ideal of $K$ over $q_{i}$. Set $\mathfrak{a}=\prod_{i} q_{i}^{e_{i}}$. Then, the ideal class of $a$ is of order $n$.

Hence, we obtain our assertion.

By Lemmas 3, 4, 5, it remains ;to construct orders $\mathfrak{D} \in M(p, 1)$ with $n_{0}=n$ when $(p, n)=(2,11),(2,13),(2.17),(2,19),(2,22),(2,23)$.

Using the table of Wada [9], we see, by a simple calculation, that the maximal order of the following imaginary quadratic field $K(p, n)$ is an example of such an order for the above $(p, n)$.

\begin{tabular}{l|c|c|c}
\hline$(p, n)$ & $(2,11)$ & $(2,13)$ & $(2,17)$ \\
\hline$K(p, n)$ & $\boldsymbol{Q}(\sqrt{-167})$ & $\boldsymbol{Q}(\sqrt{-263})$ & $\boldsymbol{Q}(\sqrt{-383})$ \\
\hline$h(p, n)$ & 11 & 13 & 17 \\
\hline$(p, n)$ & $(2,19)$ & $(2,22)$ & $(2,23)$ \\
\hline$K(p, n)$ & $\boldsymbol{Q}(\sqrt{-311})$ & $\boldsymbol{Q}(\sqrt{-591})$ & $\boldsymbol{Q}(\sqrt{-647})$ \\
\hline$h(p, n)$ & 19 & 22 & 25 \\
\hline
\end{tabular}

$(h(p, n)$ denotes the class number of $K(p, n)$.)

This completes the proof of Theorem 2 . 


\section{§4. Real quadratic fields}

Set $M(p)$ (resp. $\left.M(p)_{+}\right)=\{\mathfrak{0}$; orders of imaginary (resp. real) quadratic fields with $(\mathfrak{o} / p)=1\}$. Let $N(p)$ (resp. $N(p)_{+}$) be the image of the map $\partial(p)\left(\operatorname{resp.} \partial(p)_{+}\right)$:

$$
\left.M(p) \text { (resp. } M(p)_{+}\right) \ni \mathfrak{v} \longrightarrow n_{0} \in N .
$$

By Theorem 2, $N(p)=N$. In this section, we prove the following

Proposition. $N(p)_{+}=N$.

First, we give a definition.

Definition. Let $d(>1)$ be a square free integer, and let $m(>1)$ and $g$ be natural numbers. Let $(X, Y)=(u, v)$ be a rational integral solution of the diophantine equation

$$
X^{2}-d g^{2} Y^{2}= \pm 4 m
$$

We say that $(u, v)$ is a trivial solution if $m=n^{2}$ is a square and $n \mid u$, $n \mid v g$.

LemMa 6. Let $d(>1)$ be a square free integer and $g$ a natural number. Set $K=Q(\sqrt{d})$. Let $\varepsilon=(1 / 2)(s+\operatorname{tg} \sqrt{d})$ be a nontrivial unit of the order of $K$ with conductor $g$ such that $\varepsilon>1$ and $N(\varepsilon)=-1 \quad(\operatorname{resp} . N(\varepsilon)=1$ ). For a natural number $m(>1)$, if the diophantine equation (3) has a nontrivial solution, an inequality $m \geq s / t^{2}$ (resp. $\left.m \geq(s-2) / t^{2}\right)$ holds.

When $m$ is not a square and $g=1$, this lemma was proved in Ankeny, Chowla and Hasse [2] and Hasse [3]. The proof of the general case goes through similarly and we shall not give the proof.

Now, we shall prove our proposition. Let $n$ be a natural number. We see easily that $p^{2 n}+4$ is not a square. Let $K=\boldsymbol{Q}\left(\sqrt{p^{2 n}+4}\right)$. First, we deal with the case $p \neq 2$. Write $p^{2 n}+4=g^{2} d$ with a natural number $g$ and a square free integer $d$. Let $\mathfrak{o}$ be the order of $K$ with conductor g. We claim that $(\mathfrak{o} / p)=1$ and $n_{\mathfrak{o}}=n$. We easily see that $(\mathfrak{o} / p)=1, \mathfrak{o}=$ $\left[1,\left(1+\sqrt{p^{2 n}+4}\right) / 2\right]$ and $\varepsilon=(1 / 2)\left(p^{n}+\sqrt{p^{2 n}+4}\right)$ is a nontrivial unit of $\mathfrak{D}$ with $N(\varepsilon)=-1$. Set $\alpha=1-\varepsilon$. Then, $\alpha \in \mathfrak{v}, N(a)=-p^{n}$ and $\left(\alpha, \alpha^{\prime}\right)=1$. Therefore, $\mathfrak{p}_{0}^{n}=(\alpha)$ or $\mathfrak{p}_{0}^{n}=\left(\alpha^{\prime}\right)$, hence by the definition of $n_{0}, n_{0} \mid n$. On the other hand, $p_{0}^{n_{0}}=\left(a+b\left(1+\sqrt{p^{2 n}+4}\right) / 2\right)$ for some $a, b \in Z$. Taking norms of both sides, we obtain $\pm 4 p^{n_{0}}=(2 a+b)^{2}-b^{2}\left(p^{2 n}+4\right)=(2 a+b)^{2}$ - $d g^{2} b^{2}$. Since $\left(\mathfrak{p}_{0}, \mathfrak{p}_{0}^{\prime}\right)=1,(X, Y)=(2 a+b, b)$ is a nontrivial solution of 
the diophantine equation $X^{2}-d g^{2} Y^{2}= \pm 4 p^{n_{0}}$. Therefore, by Lemma 6 and the fact that $\varepsilon$ is a unit of $\mathfrak{o}$ with $N(\varepsilon)=-1$, we get $p^{n_{0}} \geq p^{n}$, i.e. $n_{0} \geq n$. Hence $n_{0}=n$, which proves our claim. Next, we deal with the case $p=2$. Assume $n \geq 3$ and set $m=n-2(\geq 1)$. Then, $p^{2 n}+4=4 g^{2} d$ for an odd natural number $g$ and a square free integer $d$ with $d \equiv 1$ $(\bmod 8)$. We claim that for the order $\mathfrak{o}$ of $K$ with conductor $g,(\mathfrak{o} / 2)=1$ and $n_{0}=m$. Since $g$ is odd and $d \equiv 1(\bmod 8),(\mathfrak{o} / p)=1 . \quad$ Set $\alpha=$ $(1 / 2)\left(2^{n-1}+1+\sqrt{2^{2 n-2}+1}\right)$. Then, $a \in \mathfrak{D}, N(\alpha)=2^{m}$ and $\left(\alpha, \alpha^{\prime}\right)=1$. Therefore, $\mathfrak{p}_{0}^{m}=(\alpha)$ or $\mathfrak{p}_{0}^{m}=\left(\alpha^{\prime}\right)$, hence $n_{\mathfrak{o}} \mid m$. Then, similarly to the case $p \neq 2$, we see that $n_{\mathrm{o}}=m$ by Lemma 6 and the fact that $\varepsilon=(1 / 2)\left(2^{n}+2 \sqrt{2^{2 n-2}+1}\right)$ is a unit of $\mathfrak{o}$ with $N(\varepsilon)=-1$.

This completes the proof of our proposition.

Remark 1. The fact that $N(p)=N$ is also proved as follows. Let $n$ be a natural number. Set $K=\boldsymbol{Q}\left(\sqrt{1-4 p^{n}}\right)$. Write $1-4 p^{n}=g^{2} d$ for a natural number $g$ and a square free integer $d$. Then, by Lemma $2,(\mathfrak{o} / p)$ $=1$ and $n_{0}=n$, for the order $\mathfrak{o}$ of $K$ with conductor $g$.

Remark 2. We have seen that the maps $\partial(p), \partial(p)_{+}$are surjective. For any $n \in N$, the inverse image $\partial(p)^{-1}(n)$ is a finite set, but $\partial(p)_{+}^{-1}(n)$ is an infinite set. This is shown as follows.

The imaginary quadratic case: Obvious.

The real quadratic case: (The notations being as in the proof of Proposition.) First, we deal with the case $p \neq 2$. Let $(1 / 2)(s+\operatorname{tg} \sqrt{d})$ be a nontrivial unit of $\mathfrak{o}$ with $s, t>0$. Let $\mathfrak{o}_{1}$ be the order of $K$ with conductor $\left(\left(\left(p^{n}-2\right) t+s\right) / 2\right) g$. Then, we easily see that $\left(\mathfrak{o}_{1} / p\right)=1$ and $n_{a_{1}}=n$. Since there are infinitely many units of $\mathfrak{D}$, there exist infinitely many $\mathfrak{o}_{1}$ 's with $\left(\mathfrak{o}_{1} / p\right)=1$ and $n_{0_{1}}=n$. It is proved similarly when $p=2$.

Remark 3. Set $M(p, 1)_{+}=\{0$; maximal orders of real quadratic fields with $(\mathfrak{o} / p)=1\}$. Let $N(p, 1)_{+}$be the image of the map $\partial(p, 1)_{+}: M(p, 1)_{+}$ $\ni \mathfrak{D} \rightarrow n_{0} \in N$. We see that $n=1,2 \in N(p, 1)_{+}$and the inverse images $\partial(p, 1)_{+}^{-1}(1), \partial(p, 1)_{+}^{-1}(2)$ are infinite sets by considering the following real quadratic fields:

$n=1 ; K=\boldsymbol{Q}\left(\sqrt{x^{2}+4 p}\right)$ where $x$ is a rational integer prime to $2 p$. (Fields of this type were considered in Yamamoto [10].)

$n=2 ; K=\boldsymbol{Q}(\sqrt{q(q-4 p)})$ where $q$ is a prime number such that $q>$ $4 p,(-1 / q)=1$ and $(p / q)=-1$.

In view of this, we can raise questions: (1) for any $n \in N(p, 1)_{+}$, is 
the inverse image $\partial(p, 1)_{+}^{-1}(n)$ an infinite set? (2) does $N(p, 1)_{+}$coincide with $N$ ?

\section{REFERENCES}

[1] N. C. Ankeny and S. Chowla, On the divisibility of the class number of quadratic fields, Pacific J. Math., 5 (1955), 321-324.

[2] N. C. Ankeny, S. Chowla and H. Hasse, On the class number of the maximal real subfields of a cyclotomic field, J. reine angew. Math., 217 (1965), 217-220.

[ 3 ] H. Hasse, Über die mehrklassige, aber einegeschlechtige reell-quadratische Zahklkörper, Elem. Math., 20 (1965), 49-58.

[4] P. Humbert, Sur les nombres de classes de certains corps quadratiques, Comment. Math. Helv., $12(1939 / 40)$, 233-245.

[5] S.-N. Kuroda, On the class number of imaginary quadratic number fields, Proc. Japan Acad., 40 (1964), 365-367.

[6] S. Lang, Elliptic functions, Addison Wesley (1973).

[ 7 ] T. Nagel, Über die Klassenzahl imaginär-quadratischer Zahlkörper, Abh. Math. Sem. Humburg, 1 (1922), 140-150.

[8] J. B. Rosser and L. Schoenfeld, Approximate formulas for some functions of prime numbers, Illinois J. Math., 6 (1962), 64-94.

[9] H. Wada, The table of the class number of the quadratic field $\boldsymbol{Q}(\sqrt{-m}), 1 \leq m$ $<24000$, RIMS Kokyuroku, 89 (1970), 90-114.

[10] Y. Yamamoto, Real quadratic number fields with large fundamental units, Osaka J. Math., 8 (1971), 261-270.

Department of Mathematics

Faculty of Science

University of Tokyo

Hongo, Tokyo, 113 Japan 